\title{
Fission barriers of odd-mass nuclei within the HF-BCS and HTDA approaches
}

\author{
Koh Meng Hock ${ }^{1,2,3, a}$, L. Bonneau ${ }^{1,2, b}$, P. Quentin ${ }^{1,2, c}$, and H. Wagiran ${ }^{3, d}$ \\ ${ }^{1}$ University Bordeaux 1, Centre d'Etudes Nucléaires de Bordeaux-Gradignan, Chemin du Solarium, \\ 33175 Gradignan, France \\ ${ }^{2}$ CNRS-IN2P3, Centre d'Etudes Nucléaires de Bordeaux-Gradignan, Chemin du Solarium, \\ 33175 Gradignan, France \\ ${ }^{3}$ Universiti Teknologi Malaysia, 81310 Skudai, Johor, Malaysia
}

\begin{abstract}
Within two mean-field plus correlation descriptions (Hartree-Fock plus BCS or plus Highly Truncated Diagonalization Approaches) we study here some static properties of two odd-neutron nuclei $\left({ }^{235} \mathrm{U},{ }^{239} \mathrm{Pu}\right)$ from the ground-state deformation to the fission isomeric well, using three different Skyrme force parametrizations. A specific study of the polarization effects due to the account of relevant time-odd density functions is performed.
\end{abstract}

\section{Introduction}

Microscopic calculations of stationary points (stable or unstable) along the average fission paths of heavy nuclei have been calculated, over the years, through mean field approaches supplemented by some more or less crude treatments of nuclear correlations (see e.g. [1]). The latter include pairing correlations as well as those related to the restoration of some broken symmetries. Most of the efforts have been concentrated so far on the description of even-even fission barriers.

In the case of odd-mass, not to mention odd-odd nuclei, a proper evaluation of these potential energy curves is contingent upon a realistic description of polarisation effects brought in by the addition of one, or two different, nucleons as compared to the even-even case. In such unpaired systems, most of the exact time-even part of the one-body density could be treated appropriately by averaging over the contributions of both partners of the Kramers pair associated with the extra nucleonic state (as obtained in an even-even core description). This approximation is embodied within an Hartree-Fock-Bogoliubov or (-BCS) approach as the so-called Equal Filling Approximation (EFA). Calculations of the fission barriers of two odd-nuclei within this EFA framework have been already published in Refs. [2] and [3].

\footnotetext{
a e-mail: koh@cenbg.in2p3.fr

be-mail: bonneau@cenbg.in2p3.fr

ce-mail: quentin@cenbg.in2p3.fr

de-mail: husin@utm.my
}

This is an Open Access article distributed under the terms of the Creative Commons Attribution License 2.0, which permits unrestricted use, distribution, and reproduction in any medium, provided the original work is properly cited. 
A full microscopic treatment should a priori include the effects of the time reversal symmetry breaking inherent to the description of a fermionic system involving an odd-number of identical particles. Whereas specific polarization effects impacting the magnetic properties of nuclei are expected and found in such approaches (see e.g. [4]), we are mostly concerned here about their consequence on the total energy, in general, and as a function of the quantum numbers of the extra nucleon state, in particular. As functions of these quantum numbers, we are interested in the variation of the so-called specialization energies (introduced by Newton and Wheeler in 1955, to emphasize the variation of spontaneous fission half-lives occurring between even and odd heavy nuclei, see e.g. [5]). As an example, we may quote the search for the variation between the fission barrier heights and hence the fission half-lives of the ground $\left(7 / 2^{-}\right)$and isomeric $\left(1 / 2^{+}\right)$states in the ${ }^{235} \mathrm{U}$ nucleus.

Throughout this paper, we assume the validity of the axial symmetry, the intrinsic parity symmetry as well as of the Bohr-Mottelson ansatz to generate out of our intrinsic solution a nuclear state by coupling with an axial rotor further (not allowing for Coriolis coupling). This yields $I=K$ (with usual notation) in our configurations. The first assumption is clearly a crude approximation for most of the considered isotopes in the vicinity of the first barrier, while the second would be totally inappropriate in the second barrier region.

In odd-nuclei, one expects that pairing correlations are quenched with respect to what they would be in neighbouring even isotopes due to the blocking of orbitals (close to the Fermi level for ground or weakly excited nuclear states). This situation places the BCS approximation at the limit of its validity when the single particle level density around the Fermi level is weak. This is why we performed beyond the usual BCS blocking treatment, a more elaborate approach preserving the particle number named the Highly Truncated Diagonalization Approach (HTDA) [6].

\section{Theoretical considerations}

The effective Hamiltonian in use here, includes a usual Skyrme-type interaction. The added Coulomb interaction is treated exactly for what concerns its direct contribution and through the Slater approximation for its exchange part. The center of mass correction has been approximated considering only the one-body part of its kinetic energy by a renormalization of the total kinetic energy operator through a factor $\left(1-\frac{1}{A}\right)$. The Skyrme Hamiltonian density which, as shown in e.g. Refs. [7] and [8], is a function of time-even local density functions (density $\rho$, kinetic energy density $\tau$, spin current tensor $\mathbf{J}$ ) and time-odd density functions (spin density $\mathbf{s}$, current density $\mathbf{j}$, spin kinetic density $\mathbf{T}$ ).

The Hartree-Fock equations are obtained by varying the total energy with respect to the single particle states. The expression for the Hartree-Fock Hamiltonian can be found e.g. in [9] where the fields entering the Hamiltonian are functions of the local densities defined e.g. in [8]. In selecting the type of Skyrme parametrization, one can opt from either an interaction or functional point of view. From interaction point of view, one should take into account all the terms appearing in Skyrme Hamiltonian density. Otherwise, if one adopts the functional point of view one has the option of neglecting some terms in the energy functional (yet preserving some conservation laws). The Skyrme parameters have normally been fitted to even-even nuclei, such that the time-odd local densities vanishes in this case. Then, when performing calculation for odd-mass nuclei (where time-odd densities are not vanishing) one can choose between incorporating all the time-odd densities related terms or to maintain a minimal time-odd densities terms (in combination with time-even densities) to respect some symmetries. In the latter case, terms proportional to $(\mathbf{s} \cdot \triangle \mathbf{s})$ and possibly also the $\left(\mathbf{J}^{2}-\mathbf{s} \cdot \mathbf{T}\right)$ terms (depending on the conditions of the fit) are neglected. We shall refer to this as minimal time-odd densities while the inclusion of all the terms will be referred to as full time-odd densities.

We have considered three types of Skyrme parametrizations for the current study. This includes the SLyIII.xx forces [10] (xx being the value of the nuclear matter effective mass chosen to be 0.8 for the one considered here) where the spin current (tensor) density was included in the fitting process. It is 
worth noting that this force does not lead to spin (ferromagnetic) instability in contrast with some other newly fitted Skyrme forces such as TIJ [11] and SLy5 [12] parameter sets. The other two forces that we used are the $\mathrm{SkM}^{*}$ parametrization which yields good surface properties and has been fitted to the liquid drop fission barrier of ${ }^{240} \mathrm{Pu}$ [13] and the SIII parametrization [14] which provides reasonably good spectroscopic properties (see [15] and [16]).

In order to account for the pairing correlations, we have used two different approaches: the usual Bardeen-Cooper-Schrieffer (BCS) method with a seniority force and the HTDA method. In both cases, we have restricted ourselves to the $\left|T_{z}\right|=1$ (neutron-neutron and proton-proton) channels.

In the BCS case, the strength of the pairing interaction is given by the antisymmetrized matrix element between pairs of time-reversed conjugate states written as $\left\langle i \bar{i}|\hat{v}| \widetilde{j}_{\bar{j}}\right\rangle^{(q)}=-\frac{G_{0}}{11+N_{q}}$ with $G_{0}{ }^{(n)}=$ 14.5 MeV and $G_{0}{ }^{(p)}=14.66 \mathrm{MeV}$ while $N_{q}$ is the nucleon number of the q-charge state. Single particle states participating in the BCS approach were defined with a single particle energy cut-off of $6 \mathrm{MeV}$ above the Fermi level for both charge states (with a diffuse Fermi type boundary defined by a usual diffusivity parameter equal to $0.2 \mathrm{MeV}$ ).

Main features of the HTDA method can be found e.g. in Section II of Ref. [17]. The strengths of the zero range pairing interaction in the $\mathrm{T}=1$ channel only, $V_{0}^{(T=1)}$, have been fitted with respect to the oddeven binding energy differences of actinide nuclei. The retained values of $V_{0}^{(T=1)}$ for the calculations with the SIII, SkM* and SLyIII.0.8 interactions are 440, 420 and $400\left(\mathrm{MeVfm}^{3}\right)$ respectively. The manybody basis is made of single-pair, double-pair and triple-pair excitations above the quasi-vacuum state with unperturbed excitation energies, lower than or equal to three times the empirical inter-shell energy $\hbar \omega=41 A^{-1 / 3}(\mathrm{MeV})$.

\section{Results and discussions}

Calculations have been made in two approaches with respect to the time-odd terms entering the expression of the energy density: including first only minimal time-odd densities, and secondly, incorporating the full time-odd terms. To respect the conditions of the fit, however, the results for the SIII and $\mathrm{SkM}^{*}$ parametrizations reported here, are obtained with a minimal inclusion of time-odd terms in the energy density functional. The results for SLyIII.0.8 are obtained with full time-odd densities since the spin current tensor was taken into account in fitting process. The effect of including all time-odd terms for SIII is discussed in the last subsection.

The single particle eigenstates resulting from the solution of the Hartree-Fock equations have been projected onto axially symmetrical harmonic oscillator basis states with a deformation-dependent cutoff corresponding to 13 spherical major shells (see [18]).

\subsection{Ground state spectra}

Calculations of the energy spectra of the ${ }^{239} \mathrm{Pu}$ and ${ }^{235} \mathrm{U}$ nuclei with 3 different Skyrme forces has been performed (spectra not shown here). In ${ }^{239} \mathrm{Pu}$, we reproduce the experimental ground state quantum numbers $\frac{1}{2}^{+}$in all three calculations. The energy levels calculated with SLyIII.0.8 and SkM* appears to be slightly compressed as compared to the experimental levels. On the other hand, the calculated energy spectra in ${ }^{235} \mathrm{U}$ appears to be spread over a too large energy range as compared to the experiment. There are some reordering of the energy levels going from one force to another. The SkM* and SLyIII.0.8 interactions yield a $1 / 2^{+}$ground state while the first excited state $\left(7 / 2^{-}\right)$is found at $34 \mathrm{keV}$ for the former and $42 \mathrm{keV}$ for the latter. This is at variance with the experimental situation where both states are almost degenerate (the $1 / 2^{+}$state having a $76.5 \mathrm{eV}$ excitation energy). Yet, we remain clearly within the expected range of accuracy of the whole approach. 
Table 1. Heights of the first fission barrier of the ${ }^{235} \mathrm{U}$ and ${ }^{239} \mathrm{Pu}$ nuclei (in $\mathrm{MeV}$ ) corresponding to the ground state quantum numbers calculated with three Skyrme forces.

\begin{tabular}{lcrcc}
\hline Nucleus & $K^{\pi}$ & \multicolumn{1}{c}{ SIII } & SkM $^{*}$ & SLyIII.0.8 \\
\hline${ }^{235} \mathrm{U}$ & $7 / 2^{-}$ & 8.15 & 9.03 & 9.07 \\
${ }^{239} \mathrm{Pu}$ & $1 / 2^{+}$ & 10.48 & 9.48 & 10.84 \\
\hline
\end{tabular}

Table 2. Differences of specialization energies (in $\mathrm{MeV}$ ) of the the first barrier height in ${ }^{235} \mathrm{U}$ and ${ }^{239} \mathrm{Pu}$.

\begin{tabular}{ccccc}
\hline Nucleus & $K^{\pi}$ & \multicolumn{1}{c}{ SIII } & SkM $^{*}$ & SLyIII.0.8 \\
\hline \multirow{4}{*}{${ }^{235} \mathrm{U}$} & $1 / 2^{+}$ & 0.56 & -0.25 & 0.67 \\
& $5 / 2^{+}$ & -1.45 & -0.61 & -0.54 \\
& $3 / 2^{+}$ & -0.14 & - & 0.44 \\
& $7 / 2^{+}$ & -1.36 & -2.07 & -0.36 \\
\hline \multirow{2}{*}{${ }^{239} \mathrm{Pu}$} & $7 / 2^{-}$ & -0.72 & 0.40 & -0.37 \\
& $5 / 2^{+}$ & -0.92 & 0.06 & -0.65 \\
& $7 / 2^{+}$ & -0.74 & - & -0.38 \\
\hline
\end{tabular}

Table 3. Nuclear spectra in the isomeric well for the ${ }^{235} \mathrm{U}$ and ${ }^{239} \mathrm{Pu}$ nuclei. The Gogny D1S results are taken from $[2,3]$ and experimental data from [21]. Energies are given in $\mathrm{keV}$.

\begin{tabular}{ccccccc}
\hline Nucleus & $K^{\pi}$ & SIII & SkM $^{*}$ & SLyIII.0.8 & Gogny D1S & Exp. \\
\hline \multirow{4}{*}{${ }^{235} \mathrm{U}$} & $3 / 2^{+}$ & 0 & - & 0 & - & - \\
& $3 / 2^{-}$ & 102 & 0 & 404 & - & - \\
& $5 / 2^{+}$ & 365 & 208 & 420 & 0 & - \\
& $11 / 2^{+}$ & 460 & 72 & 546 & 120 & - \\
\hline \multirow{2}{*}{${ }^{239} \mathrm{Pu}$} & $5 / 2^{+}$ & 0 & 0 & 0 & 0 & 0 \\
& $9 / 2^{-}$ & -11 & 51 & 93 & 230 & 203 \\
& $11 / 2^{+}$ & 550 & 333 & 526 & 11 & - \\
\hline
\end{tabular}

\subsection{Fission barrier heights and specialization energies}

For some relevant $K^{\pi}$ values, we have performed calculations in the ground state and near the top of the first barrier. The obtained ground state fission barrier heights in ${ }^{235} \mathrm{U}$ and ${ }^{239} \mathrm{Pu}$ are shown in Table 1. The differences of specialization energies defined with respect to the ground state values are listed in Table 2. Clearly, as compared to experimental values, these barrier heights are much too high. Yet, they are overestimated in that the deformation dependence of zero-point rotational energy corrections and the effect of the triaxial instability have not been taken care of. To these sources of overestimation, one should add another slight contribution due to the relatively small basis size in use for the description of single particle states (see the discussion of [19]). On the other hand, our barrier heights are slightly underestimated due to the use of the Slater approximation for Coulomb exchange terms [20].

\subsection{Isomeric well}

We present our calculated nuclear spectra in the isomeric well in Table 3, together with the results of Ref. [2] and experimental data when available. The different Skyrme forces gives results which are reasonably consistent within themselves and when compared with available energies from the calculation of Ref. [2] and experimental data for ${ }^{239} \mathrm{Pu}$. Notably, all three forces reproduce the ordering of the energy levels in ${ }^{239} \mathrm{Pu}$ (the $5 / 2^{+}$state being the lowest, followed by a $9 / 2^{-}$state). The 
Table 4. Isomeric energy $E_{I I}$ calculated with three Skyrme forces for ${ }^{235} \mathrm{U}$ and ${ }^{239} \mathrm{Pu}$. Energies are given in $\mathrm{MeV}$. The spin and parity of the calculated (lowest) fission isomeric state are given in parenthesis.

\begin{tabular}{ccccc}
\hline & Exp & SIII & SkM $^{*}$ & SLyIII.0.8 \\
\hline${ }^{235} \mathrm{U}$ & - & $5.74\left(3 / 2^{+}\right)$ & $3.52\left(3 / 2^{-}\right)$ & $7.04\left(3 / 2^{+}\right)$ \\
${ }^{239} \mathrm{Pu}$ & $3.1\left(5 / 2^{+}\right)$ & $5.03\left(5 / 2^{+}\right)$ & $2.96\left(5 / 2^{+}\right)$ & $6.51\left(5 / 2^{+}\right)$ \\
\hline
\end{tabular}

Table 5. Heights of the first fission barrier (in MeV) obtained from HF + BCS and HTDA calculations.

\begin{tabular}{cccccccc}
\hline \multirow{2}{*}{ Nucleus } & \multirow{2}{*}{$K^{\pi}$} & \multicolumn{2}{c}{ SIII } & \multicolumn{2}{c}{ SkM $^{*}$} & \multicolumn{2}{c}{ SLyIII.0.8 } \\
\cline { 3 - 8 } & & HFBCS & HTDA & HFBCS & HTDA & HFBCS & HTDA \\
\hline \multirow{4}{*}{${ }^{235} \mathrm{U}$} & $7 / 2^{-}$ & 8.15 & 8.40 & 9.03 & 10.16 & 9.07 & 9.88 \\
& $1 / 2^{+}$ & 8.71 & 8.73 & 8.79 & 9.93 & 9.74 & 10.22 \\
& $3 / 2^{+}$ & 8.01 & 8.01 & - & - & 9.51 & 10.08 \\
& $5 / 2^{+}$ & 6.70 & 6.86 & 8.42 & 9.63 & 8.53 & 9.76 \\
& $7 / 2^{+}$ & 6.79 & 6.98 & 6.96 & 8.36 & 8.72 & 9.94 \\
\hline \multirow{2}{*}{${ }^{239} \mathrm{Pu}$} & $1 / 2^{+}$ & 10.48 & 11.12 & 9.48 & 10.71 & 10.84 & 11.46 \\
& $7 / 2^{-}$ & 9.76 & 9.93 & 9.88 & 11.26 & 10.47 & 11.06 \\
& $7 / 2^{+}$ & 9.74 & 9.74 & - & - & 10.46 & 11.68 \\
& $5 / 2^{+}$ & 9.56 & 9.52 & 9.54 & 11.05 & 10.20 & 11.35 \\
\hline
\end{tabular}

energy difference between these two levels varies slightly depending on the choice of the Skyrme parametrization. The results obtained with SLyIII.0.8 seems to agree best with experimental data, especially if one were to take into account the effect of Coriolis coupling which is about $100 \mathrm{keV}$, as indicated in the earlier work of Ref. [16]. Our calculated $11 / 2^{+}$state is much too high, at about $500 \mathrm{keV}$ above the $5 / 2^{+}$state as compared to a $11 \mathrm{keV}$ energy difference obtained in Ref. [2].

The consistency of the results obtained for ${ }^{235} \mathrm{U}$ is less manifest. The SIII and SLyIII.0.8 interactions predict $3 / 2^{+}$as the lowest energy state, while a $3 / 2^{-}$ground state is obtained with $\mathrm{SkM}^{*}$. Neither of these agree with the results of Ref. [3] which predicts a $5 / 2^{+}$state.

The lowest fission isomeric energies $E_{I I}$ are reported in Table 4 for the two nuclei for all three interactions and compared with experimental data [21] when available.

In both nuclei, the $\mathrm{SkM}^{*}$ force produced a significantly lower isomeric energy compared to the results obtained with the other two forces. It should be noted however that although the value obtained for $\mathrm{SkM}^{*}$ appears to be in good agreement with experimental data, corrections for other effects e.g. due to the rotational motion should lower the isomeric energy to unrealistic values.

\subsection{Effect of the pairing treatment with HTDA}

In order to illustrate the effect of treating the pairing part using a particle number conserving approach, we show the first barrier height obtained from HF + BCS and HTDA calculation in Table 5. In general, with the parametrization of the residual interaction in use, the effect of proper treatment of pairing results in an increase of the fission barrier height. The amount of correction depends on the choice of the Skyrme force being considered. Calculations performed with $\mathrm{SkM}^{*}$ shows a large difference between the barrier heights calculated from the two pairing approaches. For example, the barrier height of $5 / 2^{+}$ in ${ }^{239} \mathrm{Pu}$ with $\mathrm{SkM}^{*}$ calculated with $\mathrm{HF}+\mathrm{BCS}$ can be lowered as much as $1.5 \mathrm{MeV}$ compared to the HTDA solution. In fact, large differences between the barrier heights are reported for $\mathrm{SkM}^{*}$ which exceeds $1 \mathrm{MeV}$. On the other hand, the difference in the barrier height is small (less than $0.2 \mathrm{MeV}$ ) for SIII calculations with three of the four states. 


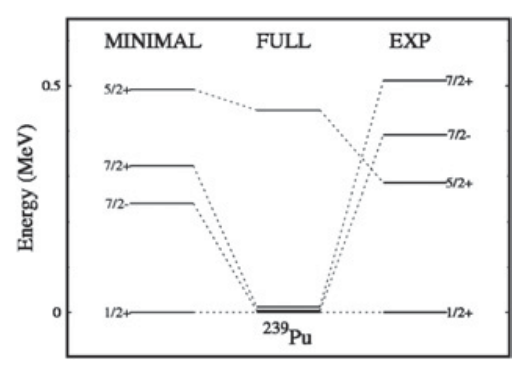

Figure 1. Ground state spectra of ${ }^{239} \mathrm{Pu}$ calculated with the SIII force with minimal and full time-odd terms as compared to experimental data.

The same effect on the difference in barrier height between HF + BCS and HTDA solution is also visible for ${ }^{235} \mathrm{U}$. Calculation with $\mathrm{SkM}^{*}$ gives the largest difference of about $1.2 \mathrm{MeV}$, while the difference for SIII is about $0.3 \mathrm{MeV}$. Calculations with SLyIII.0.8 in both nuclei gives a difference between 0.6 to $1.2 \mathrm{MeV}$.

\subsection{Effect of the neglected terms in the energy functional}

We present in this section the effect of neglected time-odd terms in the energy density functional which was not taken into account in the fitting process of the SIII and SkM* force. The results are presented for the SIII force in ${ }^{239} \mathrm{Pu}$. As can be seen from the ground state spectra of ${ }^{239} \mathrm{Pu}$ in Figure 1, the inclusion of all the time-odd terms causes the compression of the energy spectra. We have checked that the term corresponding to $(s \cdot \triangle s)$ has a negligible effect on the energy spectra, whereas the terms involving the spin-current tensor with the combination $\left(\mathbf{J}^{2}-\mathbf{s} \cdot \mathbf{T}\right)$ have a dominant effect.

We have also calculated the first fission barrier height with and without the full time-odd terms using the SIII force for ${ }^{239} \mathrm{Pu}$. In going from a minimal to a full time-odd scheme, we found a systematic lowering of the first fission barrier height of $0.95,0.72,0.54$ and $1.04 \mathrm{MeV}$ for $1 / 2^{+}, 7 / 2^{-}, 7 / 2^{+}$ and $5 / 2^{+}$respectively. At the same time, we found a lowering of $E_{A}$ by $1.0 \mathrm{MeV}$ upon using the full functional for a ${ }^{240} \mathrm{Pu}$ core, which is consistent with the above. This study shows that the absence of spin current tensor density in the original fit of the Skyrme force can be quite significant as the effect of this term on some observables-such as relative energies-cannot be absorbed in the fitted parameters.

\section{Conclusion}

We have presented here some static nuclear properties along the fission path of the ${ }^{235} \mathrm{U}$ and ${ }^{239} \mathrm{Pu}$ nuclei with three different Skyrme forces. The first fission barrier heights are overestimated in the present work. Relevant corrections will be taken into account in future work.

We have in particular shown the energy spectra at the ground state and isomeric wells. The SIII force appears to give a better agreement with the experimental data in terms of energy level spacing in the ground state. Nevertheless, the results obtained with the SkM* and SLyIII.0.8 interactions are quite reasonable.

In order to study the effect of pairing correlation from a particle number conserving approach, we have compared the fission barrier heights obtained from HF + BCS and HTDA calculations with the SIII and $\mathrm{SkM}^{*}$ interactions. Finally, we have discussed the effect of including a spin current tensor density in the energy density functional in ${ }^{239} \mathrm{Pu}$ with the SIII force. At ground state deformation, the addition of this density caused a strong compression of the energy spectra. The first fission barrier heights were 
lowered when the full time-odd densities were considered. This discussion emphasizes the importance of using effective interactions in what they have been fitted for.

\section{References}

[1] M. Bender, P.-H. Heenen and P.-G. Reinhard, Rev. Mod. Phys. 75, (2003) pp. 163-164

[2] F. de la Iglesia, V. Martin, S. Perez-Martin and L.M. Robledo, Fourth International Workshop on Nuclear Fission and Fission-Product Spectroscopy, Cadarache (France), May 13-16, 2009, eds: A. Chatillon et al., AIP Conf. Proc. 1175 (AIP, Melville, NY, 2009), pp. 199-206

[3] S. Perez-Martin and L.M. Robledo, Int. J. Mod. Phys. E 18, 788 (2009)

[4] P. Quentin, L. Bonneau, N. Minkov and D. Samsoen, Int. J. Mod. Phys. E 4, 611 (2010)

[5] John R. Huizenga and R. Vandenbosch, Nuclear Fission (Elsevier, 1973) pp. 54

[6] N. Pillet, P. Quentin and J. Libert, Nucl. Phys. A 697, 141 (2002)

[7] Y. M. Engel, D. M. Brink and K. Goeke, Nucl. Phys. A 249, 215 (1975)

[8] P. Bonche, H. Flocard and P.H. Heenen, Nucl. Phys. A 467, 115 (1987)

[9] P. Quentin, N. Minkov, L. Bonneau and D. Samsoen, ibid. Ref. [2], pp. 174-181

[10] K. Washiyama, K. Bennaceur, B. Avez, M. Bender, P.-H. Heenen and V. Hellemans, Phys. Rev. C 86, 05430 (2012)

[11] V. Hellemans, P.-H. Heenen and M. Bender, Phys. Rev. C 85, 014326 (2012)

[12] A. Pastore, D. Davesne, K. Bennaceur, J. Meyer and V. Hellemans, Phys. Scr. T154, 014014 (2013)

[13] J. Bartel, P. Quentin, M. Brack, C. Guet, H.-B.Håkansson, Nucl. Phys. A 79, 79 (1982)

[14] M. Beiner, H. Flocard, Nguyen Van Giai and P. Quentin, Nucl. Phys. A 238, 29 (1975)

[15] L. Bonneau, P. Quentin and P. Moller, Phys. Rev. C 76, 024320 (2007)

[16] J. Libert, M. Meyer, P. Quentin, Phys. Lett. B 95, 586 (1980)

[17] J. Le Bloas, L. Bonneau, P. Quentin, J. Bartel and D. D. Strottman, Phys. Rev. C 86, 034332 (2012)

[18] H. Flocard, P. Quentin, A.K. Kerman and D. Vautherin, Nucl. Phys. A 203, 433 (1973)

[19] L. Bonneau, P. Quentin and D. Samsoen, Eur. Phys. J. A 21, 391 (2004)

[20] J. Le Bloas, M.H. Koh, P. Quentin, L. Bonneau, J.I.A. Ithnin, Phys. Rev. C 84, 014310 (2011)

[21] E. Browne, Nucl. Data Sheets 98, 665 (2003) 